\title{
Correction to: A simple property of the Weyl tensor for a shear, vorticity and acceleration-free velocity field
}

\author{
Luca Guido Molinari ${ }^{1,2}$ (ID) Carlo Alberto Mantica ${ }^{2,3}$
}

Published online: 26 September 2018

(c) Springer Science+Business Media, LLC, part of Springer Nature 2018

\section{Correction to: Gen Relativ Gravit (2018) 50:81 https://doi.org/10.1007/s10714-018-2398-9}

In our paper "A simple property of the Weyl tensor for a shear, vorticity and acceleration-free velocity field" [1] the sentence: ".. a contraction with $u^{i}$ gives: $0=u^{i} \nabla_{i} E_{k m}+\varphi E_{k m}$ " in the end of the proof of Theorem 1.1 (page 4) is wrong (actually, it gives $\left.0=u^{i} \nabla_{i} E_{k m}+(n-1) \varphi E_{k m}\right)$.

The error partly changes Theorem 1.1 (stated in page 2 ) but does not affect Theorem 1.2 and all the other propositions in the paper, as well as the long evaluation in the Appendix.

The correct statement is:

Theorem 1.1 In a twisted space-time of dimension $n>3$ :

(i) $u_{m} C_{j k l}{ }^{m}=0 \Longrightarrow \nabla_{m} C_{j k l}{ }^{m}=0$

(ii) $\nabla_{m} C_{j k l}^{m}=0 \Longrightarrow u^{p} \nabla_{p}\left(u_{m} C_{j k l}^{m}\right)=-\varphi(n-1) u_{m} C_{j k l}{ }^{m}$

Proof The proof of statement (i) remains as given in page 4 of [1]. The proof of statement (ii) is as follows.

Consider the identity (8) for the Weyl tensor $C_{j k l m} u^{m}=u_{k} E_{j l}-u_{j} E_{k l}$, where $E_{k l}=u^{j} C_{j k l m} u^{m}$. Then: $u^{p} \nabla_{p}\left(C_{j k l m} u^{m}\right)=u_{k} u^{p} \nabla_{p} E_{j l}-u_{j} u^{p} \nabla_{p} E_{k l}$.

The original article can be found online at https://doi.org/10.1007/s10714-018-2398-9.

Luca Guido Molinari

Luca.Molinari@unimi.it

Carlo Alberto Mantica

Carlo.Mantica@mi.infn.it

1 Physics Department, Università degli Studi di Milano, Via Celoria 16, 20133 Milan, Italy

2 I.N.F.N. Sez. Milano, Via Celoria 16, 20133 Milan, Italy

3 I.I.S. Lagrange, Via L. Modignani 65, 20161 Milan, Italy 
If $\nabla^{m} C_{j k l m}=0$ Eq. (15) holds, i.e. $u^{p} \nabla_{p} E_{i j}=-\varphi(n-1) E_{i j}$. Then:

$$
u^{p} \nabla_{p}\left(C_{j k l m} u^{m}\right)=-\varphi(n-1)\left(u_{k} E_{j l}-u_{j} E_{k l}\right)=-\varphi(n-1) C_{j k l m} u^{m}
$$

In the special case of generalised Robertson-Walker space-times the original statement $u_{m} C_{j k l}{ }^{m}=0 \Longleftrightarrow \nabla_{m} C_{j k l}{ }^{m}=0$ remains true (Theorem 3.4 of Ref. [2]).

\section{References}

1. Molinari, L.G., Mantica, C.A.: A simple property of the Weyl tensor for a shear, vorticity and acceleration-free velocity field. Gen. Relativ. Gravit. 50, 81 (2018)

2. Mantica, C.A., Molinari, L.G.: On the Weyl and Ricci tensors of generalized Robertson-Walker spacetimes. J. Math. Phys. 57(10), 102502 (2016) 